\title{
Haptic spatial matching in near peripersonal space
}

Citation for published version (APA):

Kaas, A. L., \& van Mier, H. I. (2006). Haptic spatial matching in near peripersonal space. Experimental Brain Research, 170(3), 403-413. https://doi.org/10.1007/s00221-005-0223-7

Document status and date:

Published: 01/01/2006

DOI:

10.1007/s00221-005-0223-7

Document Version:

Publisher's PDF, also known as Version of record

Document license:

Taverne

Please check the document version of this publication:

- A submitted manuscript is the version of the article upon submission and before peer-review. There can be important differences between the submitted version and the official published version of record.

People interested in the research are advised to contact the author for the final version of the publication, or visit the DOI to the publisher's website.

- The final author version and the galley proof are versions of the publication after peer review.

- The final published version features the final layout of the paper including the volume, issue and page numbers.

Link to publication

\footnotetext{
General rights rights.

- You may freely distribute the URL identifying the publication in the public portal. please follow below link for the End User Agreement:

www.umlib.nl/taverne-license

Take down policy

If you believe that this document breaches copyright please contact us at:

repository@maastrichtuniversity.nl

providing details and we will investigate your claim.
}

Copyright and moral rights for the publications made accessible in the public portal are retained by the authors and/or other copyright owners and it is a condition of accessing publications that users recognise and abide by the legal requirements associated with these

- Users may download and print one copy of any publication from the public portal for the purpose of private study or research.

- You may not further distribute the material or use it for any profit-making activity or commercial gain

If the publication is distributed under the terms of Article $25 \mathrm{fa}$ of the Dutch Copyright Act, indicated by the "Taverne" license above, 


\section{Amanda L. Kaas • Hanneke I. van Mier \\ Haptic spatial matching in near peripersonal space}

Received: 25 April 2005/ Accepted: 5 September 2005/Published online: 23 November 2005

(C) Springer-Verlag 2005

\begin{abstract}
Research has shown that haptic spatial matching at intermanual distances over $60 \mathrm{~cm}$ is prone to large systematic errors. The error pattern has been explained by the use of reference frames intermediate between egocentric and allocentric coding. This study investigated haptic performance in near peripersonal space, i.e. at intermanual distances of $60 \mathrm{~cm}$ and less. Twelve blindfolded participants (six males and six females) were presented with two turn bars at equal distances from the midsagittal plane, 30 or $60 \mathrm{~cm}$ apart. Different orientations (vertical/horizontal or oblique) of the left bar had to be matched by adjusting the right bar to either a mirror symmetric $(/ /)$ or parallel $(/ /)$ position. The mirror symmetry task can in principle be performed accurately in both an egocentric and an allocentric reference frame, whereas the parallel task requires an allocentric representation. Results showed that parallel matching induced large systematic errors which increased with distance. Overall error was significantly smaller in the mirror task. The task difference also held for the vertical orientation at $60 \mathrm{~cm}$ distance, even though this orientation required the same response in both tasks, showing a marked effect of task instruction. In addition, men outperformed women on the parallel task. Finally, contrary to our expectations, systematic errors were found in the mirror task, predominantly at $30 \mathrm{~cm}$ distance. Based on these findings, we suggest that haptic performance in near peripersonal space might be dominated by different mechanisms than those which come into play at distances over $60 \mathrm{~cm}$. Moreover, our results indicate that both inter-individual differences and task demands affect task performance in haptic spatial matching. Therefore, we conclude that the study of haptic spatial matching in near peripersonal
\end{abstract}

A. L. Kaas $(\bowtie) \cdot$ H. I. van Mier

Faculty of Psychology, Department of Cognitive Neuroscience, Universiteit Maastricht, P.O. Box 616 ,

6200 Maastricht, The Netherlands

E-mail: a.kaas@psychology.unimaas.nl

Tel.: + 31-43-3882172

Fax: + 31-43-3884125 space might reveal important additional constraints for the specification of adequate models of haptic spatial performance.

Keywords Human - Egocentric/allocentric $\cdot$ Reference frames $\cdot$ Gender

\section{Introduction}

For successful interaction with objects in peripersonal space, an accurate representation of this space seems indispensable. However, evidence indicates that both visual (Cuijpers et al. 2000; Hermens and Gielen 2003; Bingham et al. 2004; Henriques et al. 2005) and haptic (Kappers 1999, 2002, 2004; Kappers and Koenderink 1999; Luyat et al. 2001; Newport et al. 2002; Henriques et al. 2004) spatial representations are prone to systematic errors. When participants are instructed to make line segments parallel to each other using active touch (haptic parallel matching), the error pattern indicates that what is perceived as parallel systematically deviates from what is physically parallel (Kappers 1999, 2002, 2004; Kappers and Koenderink 1999; Newport et al. 2002; Zuidhoek et al. 2003). Counter clockwise errors are performed when the orientation of bars to the left of the midsagittal plane are to be made parallel to a bar at a location to the right. Conversely, clockwise errors occur when making bars to the right parallel to bars to the left of the midsagittal plane (Kappers 2003, 2004). The magnitude of the errors increases at greater distances between the bars (Kappers 1999, 2002; Kappers and Koenderink 1999; Zuidhoek et al. 2003, 2004).

It might be argued that errors in haptic spatial matching result from a lack of sensitivity or acuity in the haptic domain. However, research by Henriques and Soechting (2003) suggests that haptic perception is comparable to visual perception in terms of acuity, stressing the importance and sensitivity of the haptic system. In spite of haptic acuity, systematic errors are observed in haptic spatial matching tasks. This indicates 
that the errors arise from non-veridical haptic spatial representations which guide task execution. Hermens et al. (in press) had participants report the orientation of haptically perceived bars as a clock time, and found that the errors were much smaller than the errors on a haptic parallel matching task. In addition, she found that the errors made when turning bars to a predefined orientation were also much smaller than haptic parallel matching errors. Hermens et al. concluded that haptic matching errors do not originate in the haptic perception or production of orientations, but rather in the transfer of the perceived bar orientation to the location of the test bar.

The analysis of systematic error patterns has been used in attempts to reveal the reference frames in which these internal representations are coded (McIntyre et al. 2000; Carrozzo et al. 2002). The directionality of the error pattern in haptic spatial matching as described above has been hypothesized to reflect the influence of an egocentric reference frame. Egocentric reference frames are centred on, or refer to the participant's body, as opposed to allocentric reference frames, which are linked to extra-bodily cues, like the direction of the gravitational pull or (haptic) contextual cues (Batista 2002; Luyat et al. 2001; see Fig. 1).

However, although the use of an egocentric reference frame explains the directional bias of the haptic parallel matching errors very well, it does not explain the size of the errors. In other words, the size of the errors which are empirically observed is smaller than to be expected when a purely egocentric frame would be used. Therefore, haptic spatial matching performance has been proposed to be the product of a reference frame intermediate between an allocentric frame, fixed in external space, and an egocentric frame, centred on the midsagittal plane (Flanders and Soechting 1995; Kappers 2003, 2004). Although this explanation proved to be very powerful in describing global error patterns and individual differences, the fact that the magnitude of the errors in haptic parallel matching tasks is not the same for different orientations still remains unexplained. Vertical and horizontal (cardinal) orientations are usually more accurately processed than oblique orientations. This so called 'oblique effect', first described by Appelle (1972), is observed in the precision (variability) of the responses and the average error size in both visual and haptic tasks, in adults as well as in infants, children, and adolescents (Gentaz and Hatwell 1995, 1998; Flanders and Soechting 1995; Kappers 1999, 2002, 2003, 2004; Gentaz et al. 2001; Luyat et al. 2001; Cuijpers et al. 2003; Luyat and Gentaz 2002; Van Mier et al. 2003; Gentaz and Streri 2004; Fraussen et al 2004; Fernandez and van Mier 2005).

The extent to which haptic performance is dominated by egocentric representations is participant-dependent, as indicated by great inter-individual variation in error size on the parallel matching task. The variation could not be accounted for by factors such as arm length, shoulder width, familiarity with or specific training on
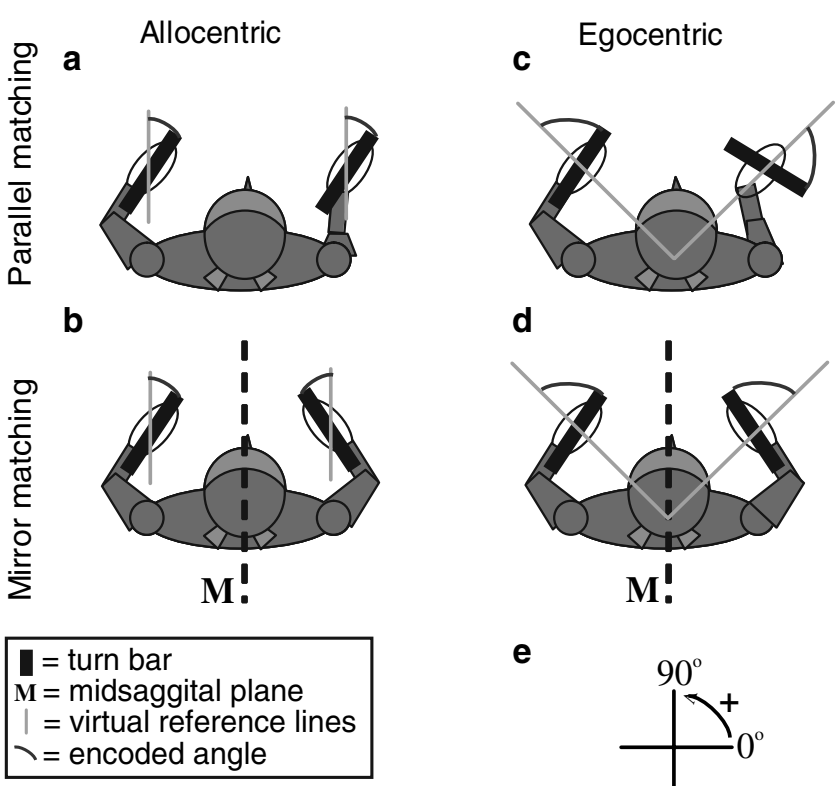

e

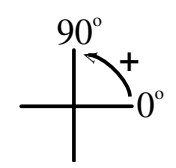

Fig. 1 Schematic overview of the parallel and mirror task and hypothetical responses in a purely allocentric or purely egocentric reference frame. The participants' hands have been made transparent to allow viewing of the turn bars. Grey lines indicate imaginary reference lines through the centre of the turn bar, originating from the participant's ego centre or parallel to allocentric reference frame axes. The reference line corresponds to the axis through the pivot of the turnbar that runs parallel to the participant's midsagittal plane in the allocentric reference frame, whereas it corresponds to the line through the participant's ego centre and the pivot of the turnbar in the egocentric reference frame. As opposed to the parallel task, performance of the mirror task requires different processing of the encoded angle. In the mirror task, the angle has to be subtracted from the orientation of the reference line through the pivot of the turnbar, whereas it needs to be added in the parallel task. a Parallel matching would be veridical in a purely allocentric frame. b However, in an egocentric reference frame, parallel matching would show strong deviations, correlated to the natural position of the hand at the location of the test bar, (c) and (d) whereas mirror matching would be veridical in both an allocentric and an egocentric frame. e Definition of the $0^{\circ}$ orientation and the sign convention used in this study

the task (Kappers 2003, 2004: personal communication). However, error size was significantly correlated with gender and profession. In other words, male technicians were better at haptic parallel matching than females with administrative jobs, but, interestingly, male physicists also had smaller deviations than female physicists (Kappers 2003). These findings suggest that the weighting factor for the contribution of the egocentric reference frame might be variable across participants and different between males and females, but stable across different tasks and conditions.

If the proposed intermediate frame is indeed used in haptic spatial matching tasks, we would expect an average error around zero when the participant's task is to produce the mirror image of a haptically explored line orientation in the midsagittal plane, because this task can be performed accurately in both reference frames that contribute to the intermediate frame (see Fig. 1b, 
d). Additionally, one would expect that distance between the hands would not affect the error size for mirror symmetry matching. With respect to the former, in a study in which participants performed both a mirror and a parallel matching task, Kappers (2004) actually found an average error of zero for mirror symmetry matching at $120 \mathrm{~cm}$. However, in a mirror symmetry experiment at $20 \mathrm{~cm}$ distance, Newport et al. (2002) found clockwise and counter clockwise errors, depending on the orientation of the bar. The errors reported for parallel matching did follow the expectations based on the intermediate reference frame model. Note that Newport's analyses used signed errors, in which any residual perceptual or executive error would cancel to zero, due to its normal distribution around the veridical response. Therefore, no directed errors could have arisen, had the participants' behaviour been guided by the intermediate frame proposed by Kappers, and these unexpected results indicate that different mechanisms might become dominant in near peripersonal space, at distances close to the midsagittal plane. However, since parallel and mirror matching were not compared within the same participants in Newport's study, the results might also be attributed to inter-individual differences.

The present study directly compared parallel and mirror matching tasks in near peripersonal space within the same participant sample. The orientation of a bar on the left-hand side was manually explored, and subsequently matched by turning another bar at the righthand side, equidistant from the body midline. Blindfolded participants were instructed to either match the bars by making them parallel, i.e. to match the orientation in the allocentric, Cartesian coordinates of the outside world, or to create the mirror image of the orientation with respect to the midsagittal plane of the body, i.e. to match the orientation in egocentric, limbbased coordinates. Distance between the bars was either 30 or $60 \mathrm{~cm}$. Based on the predictions following from the intermediate reference frame model, we expected an effect of task (hypothesis 1), i.e. clockwise errors in the parallel task and no systematic errors in the mirror task, because the mirror task, unlike the parallel task, can be performed correctly in both an egocentric and an allocentric reference frame (see Fig. 1b, d). Besides this, we expected a task by distance effect (hypothesis 2), in the sense that the mirror task would not be affected by distance, whereas the parallel task would show a greater error size at large compared to short distance, due to the increasing mismatch between the true allocentric reference lines and the reference lines in the intermediate reference frame used when performing the task. In addition, we expected differential errors for the $90^{\circ}$ orientation in parallel and mirror matching (hypothesis 3 ), reflecting the different task instruction, even though this orientation requires exactly the same response in both tasks, and allows no variation in the positioning of the hands. Moreover, based on results from prior studies, we expected an effect of orientation (hypothesis 4) resulting in an "oblique effect" (hypothesis 5), that is, smaller average errors for the $0^{\circ}$ and $90^{\circ}$ orientation (the cardinal orientations) compared to the other, oblique orientations. Using a parallel task, Kappers $(2003,2004)$ and Van Mier et al. (2003) previously observed a between-subjects reversal in the direction of the oblique effect in participants with the highest average deviations. Those participants showed greater deviations for the cardinal orientations compared to the oblique orientations. Such a reversal would not be unlikely, considering the fact that the "oblique" orientations are defined with respect to a purely allocentric external reference frame. A participant using a more egocentric, intermediate reference frame might experience the externally defined oblique orientations as cardinal orientations. Therefore, we expected a reversal of the oblique effect for participants using a more egocentric reference frame, i.e. with the highest average errors on the parallel task, showing better performance for oblique instead of cardinal orientations (hypothesis 6). Finally, we expected an effect of gender (hypothesis 7), with male participants outperforming female participants.

The current behavioural study was carried out in relation to an fMRI study (Kaas et al. 2004) investigating the neural bases of working memory processes related to haptic orientation matching. For this purpose, a delay was introduced between the exploration and the matching phase. The introduction of a delay has previously been reported to improve parallel matching performance (Zuidhoek et al. 2003). It was found that after a $10 \mathrm{~s}$ delay, performance was significantly better than without a delay. This effect was explained by an increasing dominance of the allocentric reference frame after a delay. Hu and Goodale (2000) reported a similar positive effect in grasping movements after a delay of $5 \mathrm{~s}$. To track possible changes in performance due to a delay, delay length was varied from 5 to $10 \mathrm{~s}$. We hypothesized that a delay could potentially eliminate or decrease the difference between parallel and mirror tasks (hypothesis 8).

\section{Materials and methods}

\section{Participants}

Twelve healthy participants (average age $23 \pm 4$ years; six males, six females) were paid to participate in the present study. All participants were undergraduate students at Maastricht University and were strongly righthanded as assessed by a Dutch translation of the Edinburgh Handedness Inventory (Oldfield 1971). Informed consent was obtained prior to the experiment. Participants had never seen the set-up, were naïve with respect to the stimulus orientations, and were unaware of the objectives of the present study. Participants wore headphones and were blindfolded to prevent visual input from influencing task performance. The study was approved by the local ethics committee and was performed in accordance with the declaration of Helsinki of 1964. 
Apparatus and stimuli

Two turn bars $\left(10 \times 1 \mathrm{~cm}^{2}\right)$ with a blunt plastic needle at each end were mounted on a table. The orientation of the bars could be read with an accuracy of $0.5^{\circ}$. The distance between the pivots of the bars was $30 \mathrm{~cm}$ (small) or $60 \mathrm{~cm}$ (large), depending on the experimental condition. The participant was seated on a chair, of which the legs were placed at a fixed position with respect to the table, to ensure that the body symmetry plane coincided with the plane that orthogonally bisected the line between the pivots of the bars. Five participants performed the small distance condition as part of an fMRI experiment (Kaas et al. 2004). They lay supine in the scanner, their heads and arms stabilized with foam padding. A small table was placed over the scanner bed below the waistline, with the two turn bars at $35 \mathrm{~cm}$ distance on top. We found no significant differences between participants performing the parallel and mirror task at the small distance condition in or out of the scanner. For reasons of simplicity, we will refer to this distance also as $30 \mathrm{~cm}$ in the remainder of the paper.

\section{Procedure}

Participants were blindfolded throughout practice and experimental trials. They were instructed to feel the orientation of a reference bar positioned to the left of the body with the left hand. After a variable delay, a test bar had to be turned with the right hand to an orientation that matched the orientation of the reference bar. A different matching criterion was used in the two experimental conditions. In the parallel condition, the test bar had to be turned to an orientation parallel to the reference bar. In the mirror condition, the test bar had to be turned to coincide with the mirror image of the left bar with respect to the midsagittal plane of the body. Figure 1 shows a schematic overview of the set-up for both tasks. Each trial was composed of an exploration phase $(1,500 \mathrm{~ms})$, a delay phase (short $(500 \mathrm{~ms})$, intermediate $(5,000 \mathrm{~ms})$ or long $(10,000 \mathrm{~ms})$ delay), and a response phase $(2,000 \mathrm{~ms})$. Start and end of the exploration and response phases were indicated by auditory signals presented through headphones (pure tones, $1,000 \mathrm{~Hz}$ for the exploration phase and $2,000 \mathrm{~Hz}$ for the response phase). Participants were instructed to remove their left hand from the reference bar at the end of the exploration phase and return it to their body. The same procedure was applied regarding the right hand at the end of the response phase. The only explicit instruction given to the participants concerning the starting and return position of the hands was that they should touch neither the table nor the bars. In practice, most participants kept their hands in the same resting position at breast height next to their torso throughout the experiment. This allowed them to start exploring or matching as soon as the respective auditory signals sounded.
Before each trial, the experimenter positioned the reference bar in one of six predetermined orientations $\left(0^{\circ}, 30^{\circ}, 60^{\circ}, 90^{\circ}, 120^{\circ}\right.$ and $150^{\circ}$, see Fig. 2$)$ while the test bar was positioned at either $55^{\circ}$ or $125^{\circ}$, varying across runs. After the response, the experimenter read the orientation indicated by the needle attached to the test bar, and wrote it down. Participants performed a total of eight runs, two for each combination of task and distance. Each run was made up of a pseudo-random series of 36 trials ( 3 delays $\times 6$ orientations $\times 2$ repetitions) of either the parallel or mirror task at 30 or $60 \mathrm{~cm}$ distance, resulting in a total of 288 trials. The order of conditions was counterbalanced over participants. Participants had practiced both tasks prior to the experiment, and could perform exploration and response within the time indicated by the auditory signals.

\section{Data analyses}

The orientation parallel to the midsagittal plane was defined as $90^{\circ}$, and the counter clockwise direction as positive. Signed errors were calculated for every trial by subtracting the orientation of the (left) reference bar from the orientation of the (right) test bar. In the mirror condition, matching errors were expressed as deviations from the mirrored reference orientation (i.e. $60^{\circ}=>120^{\circ}$ ). Errors were subsequently normalized to lie between $-89^{\circ}$ and $90^{\circ}$ and averaged over runs.

The errors were analyzed in a 2 (Task: parallel/mirror $) \times 2$ (Distance: $30 / 60 \mathrm{~cm}) \times 6$ (Orientation: $0^{\circ}, 30^{\circ}$, $60^{\circ}, \quad 90^{\circ}, \quad 120^{\circ}$, and $\left.150^{\circ}\right) \times 3$ (Delay: $500 / 5,000 /$ $10,000 \mathrm{~ms}$ ) within-subjects repeated measurement ANOVA, with gender as between-subjects factor. In an additional analysis, the six different orientations were clustered in two categories: $0^{\circ}$ and $90^{\circ}$ trials were averaged and entered as cardinal orientations, and all other orientations were aggregated to one oblique variable, resulting in a within-factor Obliqueness with two levels.

An additional analysis was performed on the variability of the responses, using a delay (3) by task (2) by distance (2) by orientation (6) repeated measures GLM with gender as between factor, and a delay (3) by task (2) by distance (2) by obliqueness (2) repeated measures GLM with gender as between factor.

\section{Results}

Hypothesis 8, concerning the effect of delay was not confirmed. There was no significant main effect of delay on subsequent matching error $(P<0.148)$, nor were there any significant interactions with delay. We therefore averaged over Delay. Unless otherwise indicated, all $P$ values reported in the following paragraph originate from the subsequent 2 (Task: parallel/mirror) $\times 2$ (Distance: $30 / 60 \mathrm{~cm}) \times 6\left(\right.$ Orientation: $0^{\circ}, 30^{\circ}, 60^{\circ}, 90^{\circ}, 120^{\circ}$, and $150^{\circ}$ ) ANOVA, with gender as between-subjects 


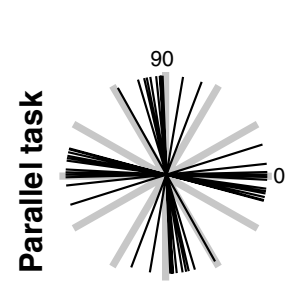

Distance $30 \mathrm{~cm}$
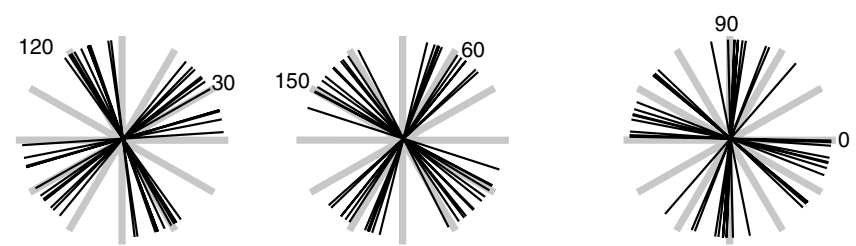

Distance $60 \mathrm{~cm}$

reference orientation

- mean matching response
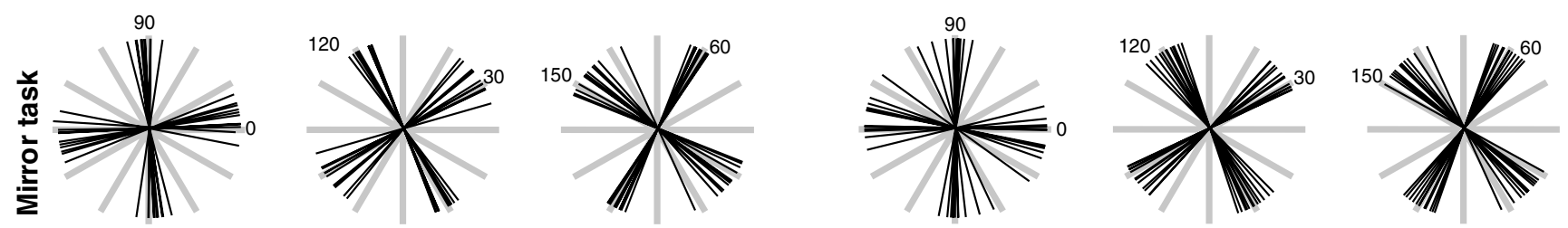

Fig. 2 Mean responses of all participants for each orientation, task and distance. To allow the reader to discriminate the responses for each individual reference orientation, each panel shows the responses for two reference orientations separated by at least $60^{\circ}$

factor. Figure 2 shows the mean responses from individual subjects for each task, distance and orientation.

Effects of task, distance and orientation

A significant main effect was found for Task ( $F(1$, $10)=16.0, P<0.003)$, confirming hypothesis 1 . Signed error was smaller for mirror versus parallel matching. The parallel task showed clockwise errors of $11^{\circ}$ on average, whereas the mirror task was characterized by an average error of $1^{\circ}$ clockwise.

Hypothesis 2, concerning the interaction between task and distance was also confirmed. There was a significant interaction $(F(1,10)=26.8, P<0.000)$, with the distance effect being most pronounced in the parallel task. Errors during parallel matching increased on average from $2^{\circ}$ clockwise to $17^{\circ}$ clockwise when the distance between the bars was changed from 30 to $60 \mathrm{~cm}$, while the errors during mirror matching on average increased from $2^{\circ}$ counter clockwise at $30 \mathrm{~cm}$ to $3^{\circ}$ clockwise at $60 \mathrm{~cm}$. Distance also showed a main effect $(F(1,10)=75.2, P<0.000)$. In general, increasing the distance between the bars from 30 to $60 \mathrm{~cm}$ was associated with an error increase of an average $10^{\circ}$ in clockwise direction. Counter clockwise errors occurred for mirror matching at a distance of $30 \mathrm{~cm}$, whereas parallel matching at the same distance lead to small clockwise errors. For the $0^{\circ}(t(11)=2.5), 30^{\circ}(t(11)=2.3)$ and $90^{\circ}(t(11)=2.5)$ orientations, these counter clockwise mirror matching errors differed significantly from zero, as demonstrated by one-sided $t$-tests with an alpha of $5 \%$.

Hypothesis 3, regarding the expected task effect for $90^{\circ}$ trials, was only confirmed for the $60 \mathrm{~cm}$ distance $(F(1,10)=5.1, P<0.047)$. This was due to the interaction of Task by Distance which was also significant in the separate analysis including only the $90^{\circ}$ trials
$(F(1,10)=10.7, P<0.008)$. At $60 \mathrm{~cm}$ distance, parallel matching of the $90^{\circ}$ orientation was associated with an average error of $9^{\circ}$ in clockwise direction, which was significantly greater than the $2^{\circ}$ counter clockwise error observed for mirror matching of the same orientation. By contrast, at a distance of $30 \mathrm{~cm}$ between the bars, both parallel and mirror matching of the $90^{\circ}$ orientation induced a counter clockwise error: $6^{\circ}$ for parallel matching and $4^{\circ}$ for mirror matching, resulting in a nonsignificant difference $(F(1,10)=0.231, P<0.641)$.

The significant main effect for Orientation $(F(5$, $50)=12.7, P<0.000)$ confirmed the fourth hypothesis. When looking at the average across tasks and distances, orientations of $120^{\circ}$ and $150^{\circ}$ resulted in the largest deviations, while $30^{\circ}$ and $90^{\circ}$ were performed most accurately. Orientation interacted with both Task $(F(5$, $50)=4.0, \quad P<0.004)$ and Distance $(F(5,50)=2.9$, $P<0.022$ ) (see Fig. 3). The task effect was largest at $30^{\circ}$ and smallest at $90^{\circ}$, with $17^{\circ}$ and $5^{\circ}$, respectively. The distance effect was largest at $0^{\circ}\left(15^{\circ}\right)$ and smallest at $150^{\circ}$ $\left(4^{\circ}\right)$. In addition, there was a three-way Task $\times$ Distance $\times$ Orientation interaction $(F(5,50)=4.7, P<0.001)$.

\section{Effect of obliqueness}

In line with our fifth hypothesis, the analysis regarding the separate orientations assigned to the categories cardinal or oblique, showed that oblique orientations generally yielded more clockwise (negative) deviations than oblique orientations $(F(1,10)=11.6, P<0.007)$ with an average deviation of $3^{\circ}$ clockwise for cardinal and $7^{\circ}$ clockwise for oblique orientations (see Fig. 4). However, in the sense of absolute error sizes, we observed a (within-subjects) reversal of the direction of the oblique effect in the mirror task compared to the parallel task. By this, we mean that cardinal orientations in the mirror task showed a greater error than oblique orientations, 


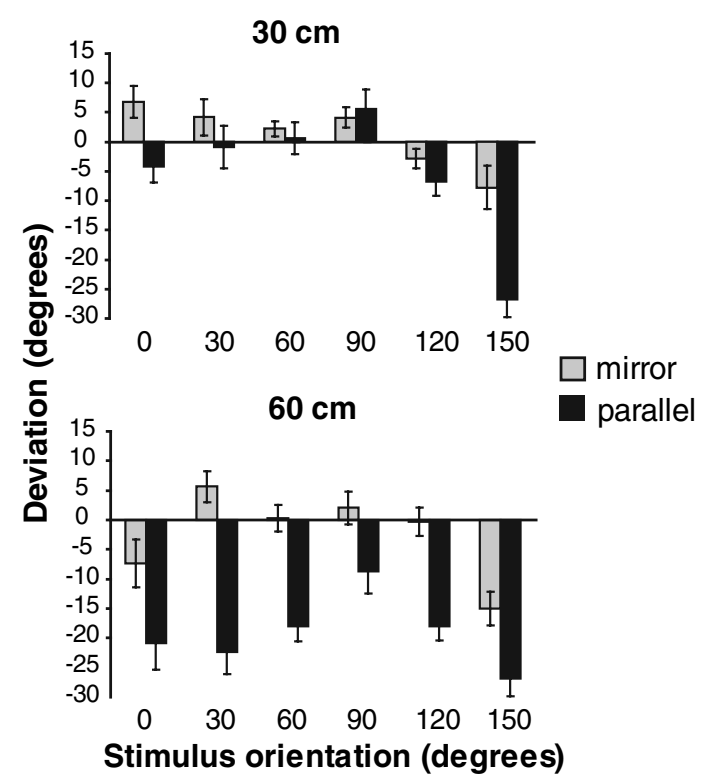

Fig. 3 Mean signed error for each task, intermanual distance and reference bar orientation. Error bars represent the standard error of the mean. Mean signed error was calculated by subtracting the orientation of the left reference bar from the orientation of the right test bar

especially at the $30 \mathrm{~cm}$ distance. At this distance, an error of $5^{\circ}$ was observed in the counter clockwise direction for cardinal orientations, and of $1^{\circ}$ clockwise for oblique orientations. At $60 \mathrm{~cm}$, the cardinal error for mirror matching was $3^{\circ}$ clockwise, which was only slightly bigger than the $2^{\circ}$ clockwise oblique error. By contrast, in the parallel task, increasing the distance between the bars by $30 \mathrm{~cm}$ caused significantly larger clockwise errors for both cardinal and oblique orientations. This resulted in a Task $\times$ Distance $\times$ Obliqueness interaction $(F(1,10)=9.4, P<0.012)$.

As can be seen in Fig. 5, hypothesis 6 was not confirmed. We did not observe a reversal of the oblique effect for participants with the highest overall average error. Almost all participants showed greater deviations

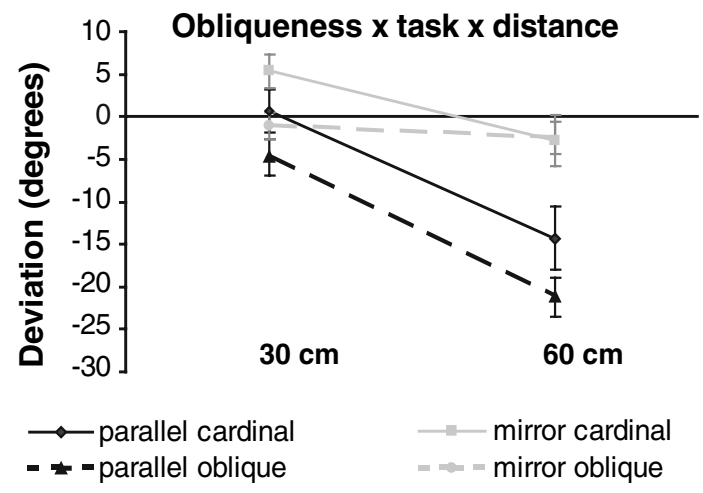

Fig. 4 Mean signed errors and standard errors displayed for task, intermanual distance, and cardinal or oblique reference bar orientations for the oblique compared to the cardinal orientations in the parallel task. However, fitted linear trend lines confirmed that the difference between the errors for cardinal and oblique orientations was smaller for participants with larger average errors. When interpolating the fitted linear trend lines for the parallel task at $60 \mathrm{~cm}$, the oblique effect would be expected to reverse for participants with an average error over approximately $-38^{\circ}$ as can be seen in Fig. 5.

\section{Effect of gender}

Hypothesis 7, concerning the better performance of male participants, was confirmed. On average, men outperformed women on both haptic matching tasks $(P<0.032)$, with deviations of $2^{\circ}$ clockwise for men and $10^{\circ}$ clockwise for women. There was a trend towards a Gender $\times$ Task interaction $(P<0.066)$, indicating that the effect of gender was most pronounced for parallel matching, where average errors of $5^{\circ}$ clockwise were observed for male participants versus $17^{\circ}$ clockwise for female participants (see Fig. 6).

\section{Precision effects}

In the additional analysis on the variation of the responses, using a delay (3) by task (2) by distance (2) by orientation (6) repeated measures GLM with gender as between factor, we found significant main effects for orientation and delay, and significant interactions for orientation by gender, delay by distance, task by gender, and orientation by distance by gender. The main effect for orientation $(F(5,50)=2.734, P<0.029)$ resulted

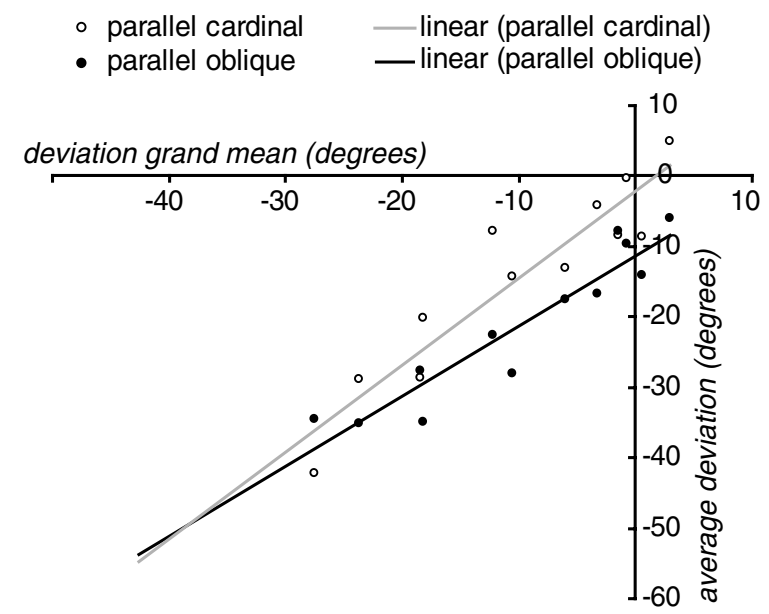

dev. cardinal $=1.2379 x-2.0481 ; R^{2}=0.8631$

dev. oblique $=0.9956 x-11.365 ; R^{2}=0.8869$

Fig. 5 Each participant's average signed error for parallel matching of cardinal and oblique orientations at $60 \mathrm{~cm}$ intermanual distance, plotted against his/her overall average signed error, and the trend lines fitted through these data points 


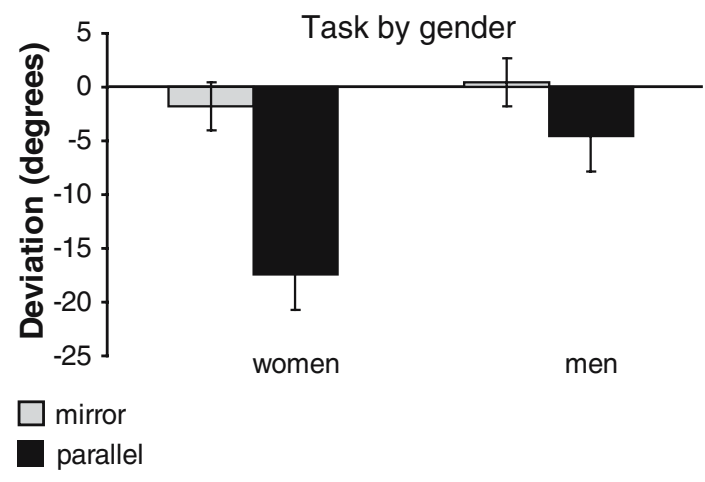

Fig. 6 Mean signed error and standard error for each task and gender

from a significantly lower variability for the $90^{\circ}$ orientation, whereas the means for the other five orientations did not differ from each other. Orientation interacted with gender $(F 5,50)=2.597, P<0.036)$, in the sense that apart from the $90^{\circ}$ orientation, the $0^{\circ}$ orientation was also associated with lower variability in male participants. We found a main effect of delay $(F(2,20)=4.890$, $P<0.019$ ), due to the fact that the short delay of $500 \mathrm{~ms}$ was associated with the lowest variability in the error, whereas the intermediate (5s) and long (10 s) delay showed a similar, higher variability. The effect of delay interacted with distance $(F(2,20)=3.896, P<0.037)$. Delay caused a greater increase in the variability at the largest $(60 \mathrm{~cm})$ distance. Although there was no main effect for task, there was a task by gender interaction $(F(1,10)=17.523, \quad P<0.002)$ : while variability was approximately equal in males and females for the parallel task, men showed a slight decrease in variability for the mirror task, whereas women showed a slight increase for the mirror task, compared to the parallel task. The interaction between orientation, distance and gender $(F(5,50)=2.963, P<0.02)$ was caused by the fact that men and women showed different orientation-dependent patterns of variability at the largest distance. Whereas the locations of minimum and maximum variability were very similar at $30 \mathrm{~cm}$ intermanual distance, the locations were shifted by approximately $30^{\circ}$ at the $60 \mathrm{~cm}$ distance. Men showed the lowest variability for the $0^{\circ}$ and $60^{\circ}$ orientations and the highest for $30^{\circ}$ and $150^{\circ}$ orientations, while women were most precise for $30^{\circ}$ and $90^{\circ}$ orientations and least precise for $0^{\circ}$ and $60^{\circ}$. Please note that we did not find an oblique effect in the precision of the responses in analyses where we categorized orientations as oblique or cardinal (delay $\times$ task $\times$ distance $\times$ oblique $\times$ gender analysis: $F(1,10)=0.061$ $(P<0.810)$ and task $\times$ distance $\times$ oblique $\times$ gender analysis: $F(1,10)=2.619(P<0.137))$.

Although the $90^{\circ}$ orientation was associated with the lowest variable error, we did not find an oblique effect when performing a delay (3) by task (2) by distance (2) by obliqueness (2) repeated measures GLM with gender as between factor, nor did we find an oblique by gender interaction.

\section{Discussion}

The present study examined the effect of instruction on haptic matching performance. Blindfolded participants were instructed to rotate a test bar with their right hand in such a way that it paralleled or mirrored the orientation of a reference bar, which could appear in different orientations and was felt with the left hand. Intermanual distance, i.e. distance between the reference and test bars, was either 30 or $60 \mathrm{~cm}$. In accordance with hypothesis 1, we found an effect of Task: participants made significantly smaller errors in the mirror task compared to the parallel task. In addition, we also found a significant Task by Distance interaction. Parallel matching performance was characterized by large clockwise errors, which increased at greater intermanual distance and when oblique orientations had to be matched. Mirror matching performance induced small clockwise errors at $60 \mathrm{~cm}$, whereas it induced counter clockwise errors for some orientations at $30 \mathrm{~cm}$ distance between the bars. For the $90^{\circ}$ trials, we found a task effect at $60 \mathrm{~cm}$, but not at $30 \mathrm{~cm}$. In line with hypothesis 4 , orientation of the reference bar had a significant effect on error size. Orientation was found to interact with Task and with Distance. As hypothesized, there was a gender effect. Male participants outperformed female participants, an effect which was most prominent for parallel matching. Finally, Delay did not affect matching error.

\section{Effects of task, distance and orientation}

A significant task effect due to the superior performance for mirror compared to parallel matching has previously been reported by Newport et al. (2002) and Kappers et al. (2004). A performance difference between parallel versus mirror matching is especially striking if it occurs for orientations that require identical responses in both mirror and parallel task, i.e. at $0^{\circ}$ and $90^{\circ}$. At $0^{\circ}$, the participant still has the choice to position the hand with the thumb towards or away from the body. Thus, task effects on the $0^{\circ}$ trials might still be caused by motorexecutive differences, meaning that participants might have preferred to put their hands in a mirrored position (both thumbs towards or away from the body) in the mirror task, and in a parallel configuration in the parallel task. However, a variation in the positioning of the hands would not affect the $90^{\circ}$ trials, where physical limitations ensure that participants will always have their thumbs pointing towards the midsagittal plane. Therefore, in the $90^{\circ}$ trials, any remaining difference in performance most likely reflects a cognitive-computational difference between the tasks. Interestingly, Kappers (2004) indeed reported differences between deviations in both tasks at $90^{\circ}$ at $120 \mathrm{~cm}$, although she did not statistically compare mirror and parallel matching at the $90^{\circ}$ orientation. The current study ex- 
tends these results to distances within normal reaching range, reporting a significant task difference for $90^{\circ}$ trials at $60 \mathrm{~cm}$ distance.

The performance difference for identical trials in the mirror and parallel matching condition shows that our task instruction worked and that participants approached both tasks in different ways, and, more importantly, it suggests that participants used a different cognitive-computational strategy to solve both tasks, at least at $60 \mathrm{~cm}$. Adhering to the intermediate reference frame model proposed by Kappers (2004), the strategy difference could occur as a task-dependent weighting of the contributions of the allocentric Cartesian frame and the egocentric polar coordinate system fixed on the body symmetry plane. Kappers uses a similar explanation for inter-individual differences in parallel matching performance. The results from the present study, as well as other studies, indicate that reference frame weighting might also vary intra-individually, depending on task instruction (Kappers 2004; Newport et al. 2002) and/or implicit task demands, such as the availability of (noninformative) visual input (Newport et al. 2002; Zuidhoek et al. 2004) and orienting towards the test hand (Zuidhoek et al. 2004). However, the intermediate reference frame model could also explain the difference between parallel and mirror matching at the $90^{\circ}$ orientation as a result of the opposing operations performed with respect to the reference line through ego centre and test bar pivot (see Figs. 1b, d, 7b, d). To obtain the response in the parallel task, the orientation difference between the reference bar and the reference line through ego centre and reference bar pivot is subtracted from the orientation of reference line running through the test bar pivot, whereas it is added in the mirror task. Our finding that the task difference for the $90^{\circ}$ trials does not persist at $30 \mathrm{~cm}$ distance between the bars might indicate a strategy shift. Alternatively, it might be caused by a ceiling effect in the parallel matching errors, due to the fact that the detrimental effect of the egocentric reference frame diminishes at small distances between the hands.

In line with the results of the current study, Kappers et al. (2003) and Zuidhoek et al. (2003) reported significantly better performance for parallel matching at smaller distances between the bars. In terms of the intermediate reference frame model (Kappers 2003) the improvement can be interpreted to result from the fact that the virtual reference lines (Fig. 1) of the intermediate reference frame become more parallel to the Cartesian axes at smaller distances between the bars, reducing the discrepancy between what is perceived as parallel and what is truly parallel. In Kappers' intermediate reference frame model, all errors in haptic spatial matching arise from the egocentric reference frame. Since mirror matching can be performed without errors in the egocentric frame, mirror performance should not show any systematic errors and should be independent from the distance between the hands.

In addition, the intermediate reference frame model predicts that performance is independent from the ori- entation of the bars. However, a significant effect of orientation was found for mirror and parallel matching both in our study and in the study by Newport et al. (2002). Moreover, the direction of the errors was in some cases contrary to the direction of the "pull" of the egocentric reference frame, especially at the shorter distance between the bars. At $30 \mathrm{~cm}$, counter clockwise average errors occurred in the mirror task for all orientations, except $120^{\circ}$ and $150^{\circ}$. In addition, mirror matching also resulted in counter clockwise errors for $30^{\circ}$ and $90^{\circ}$ trials at $60 \mathrm{~cm}$, and for $90^{\circ}$ trials in parallel matching at $30 \mathrm{~cm}$. Therefore, adjustments to the intermediate reference frame model might be necessary to describe these variations in error direction and orientation dependency. Although the use of egocentric forelimb based axes might account for the counter clockwise errors observed for the $90^{\circ}$ orientation in parallel matching at $30 \mathrm{~cm}$, it cannot account for the fact that the parallel matching of the other five orientations lead to opposite clockwise errors at the $30 \mathrm{~cm}$ distance, whereas the reverse is true for the intermediate reference frame model.

The different error sizes for different orientations might reflect the influence of the asymmetric and anisotropic ability to rotate the hand, pulling responses towards the most natural orientation of the hand for a given arm configuration, in addition to a lower perceptual accuracy at extreme joint angles (Rossetti et al. 1994). The expected pattern of effects for parallel matching would be that orientations at increasingly counter clockwise distances from the most natural hand orientation would be encoded and reproduced with increasingly large clockwise deviations (and vice versa), on top of the general bias induced by the natural orientation of the hand. In general, the effect would be smaller in the mirror task, and would average to zero when the reference orientations are chosen in such a way that they are distributed equally over the range of all possible orientations. To some extent, this account can explain the general shape of the orientation dependency of the error in our data and in the data by Newport et al. (2002), as well as the interaction with distance, since the most natural orientation of the hand will vary at different positions with respect to the midsagittal plane. Nevertheless, it is in contradiction with the finding that the orientation effect does not average to zero in the mirror task, and with the results found by Kappers (2004), who does not report any significant systematic errors for mirror matching. Her mirror data show only small deviations in the clockwise direction, for oblique orientations when the left hand was used for exploration of the reference bar and for cardinal orientations when the right hand was used for exploration. Apart from the inclusion of trials where the right hand was used for exploration of the reference bar, the most important difference in the study by Kappers (2004) is the use of a larger intermanual distance; being $120 \mathrm{~cm}$, whereas a distance of $20 \mathrm{~cm}$ in the study by Newport et al. (2002) and 30 and $60 \mathrm{~cm}$ in the present study. However, the 
rotational abilities of the hand affect all intermanual distances. Therefore, the orientation effect is most likely caused or moderated by another factor, dependent on intermanual distance and the direction of matching (whether the left or right hand is used for exploration).

A factor that might be of importance to explain systematic errors in the mirror task is the positioning of the plane that the participant uses for the mirror operation with respect to the symmetry axes of the body, which coincided with the plane that orthogonally bisected the line between the pivots of the bars. If these planes are misaligned, systematic counter clockwise errors could arise in the mirror task. It can be derived that the overall effect of this shift is larger at small intermanual distances, because the relative discrepancy of the angles between the mirror plane and reference lines on the left and on the right becomes larger at smaller distances. This would be in agreement with the fact that the effect did not persist equally strongly at the larger distance $(60 \mathrm{~cm})$ between reference and test bars, and has only been reported in the study by Newport et al. (2002) using short distances. The direction of the shift might depend on the matching direction (left hand exploration, right hand response). It might also reflect an asymmetry in spatial attention, i.e. an over-representation of the left hemispace, due to right-hemispheric dominance for spatial attention. For the parallel task, Zuidhoek et al. (2003) showed that the direction of spatial attention had an effect on the error size. In our study, counter clockwise errors occurred mainly for orientations where the right hand pointed to the left.

The use of egocentric forelimb based axes might account for the counter clockwise errors observed for the $90^{\circ}$ orientation in parallel matching at $30 \mathrm{~cm}$, which cannot be accounted for by the intermediate reference frame model. On the other hand, whereas the intermediate reference frame model can account for the fact that the parallel matching of the other five orientations lead to opposite clockwise errors at the $30 \mathrm{~cm}$ distance, a model using egocentric forelimb axes could not. Both an egocentric forelimb model and the intermediate reference frame model fail to explain the systematic errors occurring in the mirror task, since both models predict error free mirror matching, because of their symmetry in the midsagittal plane of the body. Models which do not share this symmetry axis would be able to explain systematic errors in the mirror symmetry matching task. We therefore feel that it is worthwhile to include an example of such a model. Although speculative, we feel that the shifted midline model might be a plausible and elegant candidate, both from a mathematical and a neuropsychological perspective. Loosening the constraint that the centre of the reference frame should lie on the midsagittal plane of the body, it provides a oneparameter description of all phenomena explained by the intermediate reference frame model, extending it to the current observations for mirror matching in near peripersonal space.
The present study shows the need to incorporate the variability in error direction and size in models describing haptic matching for different stimulus orientations by including factors such as asymmetrical and anisotropic hand rotation as well as the potential effects of spatial attention. It might be that these factors can be accounted for by proper adjustments of the intermediate reference frame model. However, it might also be that a new model using different assumptions or a different level of description will prove more effective in the end.

Oblique effect, gender, and delay

In accordance with previous studies (Gentaz and Hatwell 1995; Kappers 1999, 2002, 2003, 2004; Gentaz et al. 2001; Luyat et al. 2001; Luyat and Gentaz 2002; Hermens and Gielen 2003; van Mier et al. 2003; Fraussen et al. 2004; Fernandez and van Mier 2005) we found an oblique effect, in the sense that the average error for vertical and horizontal (cardinal) orientations was smaller than the average error for oblique orientations. In our view, the distinction between oblique and cardinal orientations is meaningful as long as there is evidence that the orientations joined in these categories evoke similar matching responses, and behave similarly across conditions. However, this was not the case in our data. There were orientations for which the error was close to zero, but these orientations were not necessarily cardinal. A change in the distance between the bars also changed the ranking order of the orientations with respect to the error size. Most previous studies have tested at intermanual distances of $60 \mathrm{~cm}$ or more, using oblique orientations of $45^{\circ}$ and $135^{\circ}$, whereas we tested at 30 and $60 \mathrm{~cm}$, using $30^{\circ}, 60^{\circ}, 120^{\circ}$, and $150^{\circ}$. We only found a special role for the $90^{\circ}$ orientation, parallel to the body midline, independent of the distance between the bars and hands. For that reason, it may be important to keep in mind that the cardinal/oblique distinction is allocentrically defined. It seems very likely that what is experienced as oblique or cardinal is participant dependent, linked to participant-specific reference frames (Luyat et al. 2001; Kappers 2004). Therefore, it may be more meaningful and informative to analyse separate orientations, instead of assuming oblique and cardinal clusters a-priori.

We did not observe a reversal of the oblique effect for participants with large average errors as reported by Kappers (2003). Based on interpolation of the fitted linear trend lines, we expect that the oblique effect might reverse for potential participants with an average error over $40^{\circ}$ for the parallel task at $60 \mathrm{~cm}$ (across all orientations and both distances) in our study. Kappers (2003) reported a reversed oblique effect for participants with average errors of $57^{\circ}$ or higher. In their study, the distance between the bars $(120 \mathrm{~cm})$ was twice as big as our largest distance $(60 \mathrm{~cm})$, thus inducing larger errors. In addition, the oblique orientations $\left(45^{\circ}\right.$ and $\left.135^{\circ}\right)$ used by Kappers are different from ours. 
The gender effect observed in our study is in line with gender differences previously reported in spatial tasks (Hammer et al. 1995; Voyer et al.; 1995; Crucian and Berenbaum 1998; Parsons et al. 2004) and more specific in haptic parallelity tasks for adults, adolescents and children (Kappers 2003; Van Mier et al. 2003; Fernandez and van Mier 2005). Although sighted and blind women were reported to have better tactile acuity than men (Goldreich and Kanics 2003; van Boven et al. 2000), they apparently do not benefit from this perceptual advantage in macro geometric tasks in the somatosensory domain. The gender difference was most striking for parallel matching at $60 \mathrm{~cm}$, which might point out that women are more egocentrically biased than men.

A previous study by Zuidhoek et al. (2003) reported that a delay of $10 \mathrm{~s}$ reduced parallel matching error at 120 and $60 \mathrm{~cm}$ distances between reference and test bars. In our study, a 5 or $10 \mathrm{~s}$ delay did neither improve nor deteriorate matching error at either distance. This might be due to a ceiling effect at shorter distances, since the egocentric bias affecting parallel matching becomes smaller at smaller distances. It might also have to do with the mixed presentation of delays in the current study, given that Zuidhoek et al. (2003) used a blocked presentation of delays. The fact that delay did not result in a significant deterioration of performance, shows that memory traces and maintenance strategies for haptic stimuli were still effective at $10 \mathrm{~s}$ retention intervals, comparable to other tactile studies showing retention up to at least $15 \mathrm{~s}$ (Kiphart et al. 1992; Bowers et al. 1990; Burton and Sinclair 2000; Woods et al. 2004).

\section{Precision effects}

In our view, the effects for the precision of the responses do not fundamentally change our interpretation of the data as described in the previous paragraphs. The effect of delay can be explained as increasing noise in the representation when stored in memory. The effect of orientation parallels the effects observed for the mean errors. There was no effect of task, indicating that the two tasks are probably executed in the same reference frame. The interaction between task and gender signalizes that men and women might use a different reference frame for the mirror task. Independent of task, intermanual distance has a differential effect of the orientation-dependent pattern of precision for men and women. Women were always most precise for the $90^{\circ}$ orientation, whereas men showed a shift at the largest distance. This might indicate that women have a stronger representation of the midsagittal plane of the body.

\section{Conclusion}

Greater errors were found for parallel versus mirror orientation matching, for oblique versus cardinal orientation matching, for large versus small distances between the bars and for female versus male participants. In addition, we found a significant difference between parallel and mirror matching at an intermanual distance of $60 \mathrm{~cm}$ for the $90^{\circ}$ orientation, an orientation that in principle required the same response in both tasks. This result clearly suggests an effect of task demand.

However, for mirror matching, we found counter clockwise errors at $30 \mathrm{~cm}$ distance between the bars. The direction of these errors is not in accordance with the view that haptico-spatial information is represented in a frame intermediate between an allocentric Euclidean frame and an egocentric polar frame, centred on the midsagittal plane. Based on these findings, we suggest that haptic performance in near-peripersonal space might be dominated by different mechanisms than those that play a role at distances over $60 \mathrm{~cm}$.

Summarizing, the present study extends previous findings of systematic errors for haptic-spatial matching, and, in addition, revealed unexpected effects at small distances from the body, distances that correspond to the natural dimensions of haptic workspace. The results provide additional constraints, necessary for the specification of an adequate mathematical and neuronal model of the processes involved in haptic spatial tasks.

Acknowledgements This research was supported by a grant from the Netherlands Organization for Scientific Research (NWO \# 410-20205). We thank Kim Huiskamp for her help collecting the data.

\section{References}

Apelle S (1972) Perception and discrimination as a function of stimulus orientation: the "oblique effect" in man and animals. Psychol Bull 78:266-278

Batista A (2002) Inner space: reference frames. Curr Biol 12:R380383

Bingham GP, Crowell JA, Todd JT. (2004) Distortions of distance and shape are not produced by a single continuous transformation of reach space. Percept Psychophys 66:152-169

Bowers RL, Mollenhauer MS, Luxford J (1990) Short-term memory for tactile and temporal stimuli in a shared-attention recall task. Percept Mot Skills 70:903-913

Burton H, Sinclair RJ (2000) Attending to and remembering tactile stimuli: a review of brain imaging data and single-neuron responses. J Clin Neurophysiol 17:575-591

Carrozzo M, Stratta F, McIntyre J, Lacquaniti F (2002) Cognitive allocentric representations of visual space shape pointing errors. Exp Brain Res 147:426-436

Crucian GP, Berenbaum SA (1998) Sex differences in right hemisphere tasks. Brain Cogn 36:377-389

Cuijpers RH, Kappers AM, Koenderink JJ (2000) Large systematic deviations in visual parallelism. Perception 29:1467-1482

Cuijpers RH, Kappers AM, Koenderink JJ (2003) The metrics of visual and haptic space based on paralellity judgements. J Math Psychol 47:278-291

Fernandez I, van Mier H. (2005) Haptic perception of paralellity in adolescents and adults. 14th Conference of the european society for cognitive psychology (ESCoP)

Flanders M, Soechting JF (1995) Frames of reference for hand orientation. J Cogn Neurosci 7:182-195

Fraussen M, Kappers A, van Mier H (2004) Haptic perception of spatial relations in children and adults. Third Dutch EndoNeuro-Psycho meeting, p 244 
Gentaz E, Hatwell Y (1995) The haptic oblique effect in children's and adults' perception of orientation. Perception, 24:631-646

Gentaz E, Hatwell Y (1998) The haptic oblique effect in the perception of rod orientation by blind adults. Percept Psychophys 60:157-167

Gentaz E, Streri A. (2004) An "Oblique Effect" in infants' haptic perception of spatial orientations. J Cogn Neurosci 16:253-259

Gentaz E, Luyat M, Cian C, Hatwell Y, Barraud PA, Raphel C (2001) The reproduction of vertical and oblique orientations in the visual, haptic, and somato-vestibular systems. Q J Exp Psychol A 54:513-526

Goldreich D, Kanics IM (2003) Tactile acuity is enhanced in blindness. J Neurosci 23:3439-3445

Hammer RE, Hoffer N, King WL (1995) Relationships among gender, cognitive style, academic mayor, and performance on the Piaget water-level task. Perc Motor Skills 80:771-778

Henriques DY, Flanders M, Soechting JF (2004) Haptic synthesis of shapes and sequences. J Neurophysiol 91:1808-1821

Henriques DY, Flanders M, Soechting JF (2005) Distortions in the visual perception of shape. Exp Brain Res 160:384-393

Henriques DY, Soechting JF (2003) Bias and sensitivity in the haptic perception of geometry. Exp Brain Res 150:95-108

Hermens F, Gielen S (2003) Visual and haptic matching of perceived orientations of lines. Perception 32:235-248

Hermens F, Kappers AML, Gielen SCAM (2005). The structure of fronto-parallel haptic space is task dependent. Percept Psychophys (in press)

Hu Y, Goodale MA (2000) Grasping after a delay shifts size-scaling from absolute to relative metrics. J Cogn Neurosci 12:856-868

Kaas AL, Van Mier HI, Goebel R (2004) Working memory for manually explored object shapes: an event-related fMRI study. Neuroimage 22:S1 e998

Kappers AML (1999) Large systematic deviations in the haptic perception of parallelity. Perception 28:1001-1012

Kappers AML (2002) Haptic perception of parallelity in the midsagittal plane. Acta psychol 109:25-40

Kappers AML (2003) Large systematic deviations in a bimanual parallelity task: further analysis of contributing factors. Acta Psychol 114:131-145

Kappers AML (2004) The contributions of egocentric and allocentric reference frames in haptic spatial tasks. Acta Psychol 117:333-340

Kappers AML, Koenderink JJ (1999) Haptic perception of spatial relations. Perception 28:781-795
Kiphart MJ, Hughes JL, Simmons JP, Cross HA (1992). Shortterm haptic memory for complex objects. Bull Pyschon Soc $30: 212-214$

Luyat M, Gentaz E, Corte TR, Guerraz M (2001) Reference frames and haptic perception of orientation: body and head tilt effects on the oblique effect. Percept Psychophys 63:541-554

Luyat M, Gentaz E (2002) Body tilt effect on the reproduction of orientations: studies on the visual oblique effect and subjective orientations. J Exp Psychol Hum Percept Perform 28:10021011

McIntyre J, Stratta F, Droulez J, Lacquaniti F (2000) Analysis of pointing errors reveals properties of data representations and coordinate transformations within the central nervous system. Neural Comput 12:2823-2855

Newport R, Rabb B, Jackson SR (2002) Noninformative vision improves haptic spatial perception. Curr Biol 12:1661-1664

Oldfield RC (1971) The assessment and analysis of handedness: the Edinburgh inventory. Neuropsychologia 9:97-113

Parsons TD, Larson P, Kratz K, Thiebaux M, Bluestein B, Buckwalter JG, Rizzo AA (2004) Sex differences in mental rotation and spatial rotation in a virtual environment. Neuropsychologia 42:555-562

Rossetti Y, Meckler C, Prablanc C (1994) Is there an optimal arm posture? Deterioration of finger localization precision and comfort sensation in extreme arm-joint postures. Exp Brain Res 99:131-136

Van Boven RW, Hamilton RH, Kauffman T, Keenan JP, PascualLeone A (2000) Tactile spatial resolution in blind Braille readers. Neurology 54:2230-2236

Van Mier, HI, Blommaert J, Kappers AM (2003) Haptic perception of parallelity in children and adults. Program No. 196.18. Society for Neuroscience

Voyer D, Voyer S, Bryden MP (1995) Magnitude of sex differences in spatial abilities: a meta-analysis and consideration of critical variables. Psychol Bull 117:250-270

Woods AT, O'Modhrain S, Newell FN (2004) The effect of temporal delay and spatial differences on cross-modal object recognition. Cogn Affect Behav Neurosci 4:260-269

Zuidhoek S, Kappers AM, van der Lubbe RH, Postma A (2003) Delay improves performance on a haptic spatial matching task. Exp Brain Res 149:320-330

Zuidhoek S, Visser A, Bredero ME, Postma A (2004) Multisensory integration mechanisms in haptic space perception. Exp Brain Res 157:265-268 